\title{
The Research on the Impact of Background Music on Appetite of Custom- ers in Restaurant
}

\author{
Yang Ting*
}

Yulin University, China

\begin{abstract}
Many scholars study shows that the background music is restaurant controllable environmental factor that can affect the customer waiting time, which has some management implications for solving customer wait time problem in the service sector. We selected a restaurant around school as our experimental place. We cooperated with restaurant staff, recorded the total time of eating breakfast, lunch and dinner of 180 customers playing different types of background music (including no music, slow-paced music and fast-paced music). Finally, the experimental results and analysis are given: Background music can really affect the customer's dining time; Slow-paced background music can extend the customer waiting time, meanwhile fast-paced background music will shorten the waiting time of customers.
\end{abstract}

Keywords: Appetite, background music, customer, restaurant.

\section{INTRODUCTION}

The famous American marketing guru Philip Kilter (1973-1974) pioneered the atmosphere as a marketing concept proposed. Then a lot of restaurants introduced each element in the atmosphere into the dining environment to affect consumer sentiment and behaviour, such as colour, lighting, music, scents and visual effects. Over the past two decades, music has become an important factor in service environments [1]. About the influence of music on consumer behaviour, there are already some foreign scholars have done different studies.

In recent years, consumers are starting to pay attention to the experience of eating process, hoping to be integrated with dining and entertainment. So the service environment has become the focus of the attention of the manager [2]. The music is one of the important factors in the environment, and many foreign scholars have been focused on the research in this field for long. However, research in this field for the domestic is blank.

A restaurant's feature is that consumers enjoy dining services. So in addition to eating, consumers have focused on an experience process, hoping to get for recreation. So dining environment become the focus of consumers concerned. As an important factors in dining environment, the background music and restaurants passenger density have become the focus of marketing managers and scholars in related fields concerned. Before many scholars studied the effects of music on consumer behaviour and the effect of density on consumer behaviour. But few people at the same time taking into account the influence of the two common environmental factors on consumer behaviour in a study [3]. The scholar Harrell [4] proposed that we need to focus on the interaction of two or more environmental factors affecting the consumer [5]. Foreign research on the background music started earlier, but the number of empirical research is not much. The domestic research on scene services is more on premature.

The rest of the paper is organized as follows. In Section 2 , background music is summarized briefly. In Section 3, experimental methods and results are discussed. Finally, a conclusion is provided in Section 4.

\section{BACKGROUND MUSIC}

About define background music, a number of scholars give many definition according to its own research scenario Kari Kalinin [6] divided music into foreground music and background music according to the presence or absence of performers and other factors lyrics. Foreground music has a specific performer and lyrics. The background music is the music musicians played with no specific number of performers without words. At the same time, the background music have strict restrictions in rhythm, frequency range and volume. The Park and Young believe that the presence of lyrics had nothing to do with background music. Music used to render the atmosphere can be called background music.

On abroad a survey showed [7]: 52 different types of managers in retail outlets, identification background music can lead consumers to buy more accounted for $76 \%$, and recognized that music has a positive impact on consumer sentiment accounted for $82 \%$. 560 consumers, 70 present of consumers prefer shops playing background music. But these judgments of the managers are not reliable data derived from the survey results, but with faith and intuition.

There is Millikan study on the background music [8]: With background music as the independent variable, by no background music, slow background music and background music fast three operating modes, in a medium-sized super- 
market do an experiment. He observed that the slow-paced background music will slow down the rate of flow of consumers in the store, at a slower pace, stay longer and produce a higher amount of consumption. The fast-paced background music will cause consumers to accelerate the flow velocity in the store, and the amount of consumption is low. When consumers are shopping in the store, the background music may subconsciously influence their buying behaviour. The effect of music rhythm on people is most obvious. Rhythm soft and slow music gives a calm, safe feeling. If the rhythm of the music is too strong it may bring feelings of irritability and anxiety.

Although these studies have proved that there is a positive relationship between the music speed and the emotions. It seems that faster music will bring more positive emotional state. But there is also more powerful evidence proved that this relationship is not one-way. It is likely an inverted Ushaped function. There is a strong correlation between the pitch and the happiness. Western culture thinks that most tuning express the vibrant positive emotions. Only a few tuning is used to convey the opposite negative feelings. These results support the traditional knowledge: A few tune is used to express sad, angry or some mysterious qualities; In comparison terms, most tuning is used to show happiness, fresh or happy emotional state.

Another aspect related with the rhythm is music speed. In the past few years, some scholars researching the speed of the music's impact on people's emotions got the same conclusion: Under the same other conditions, slow music will make people feel more cheerful and pleasant than fast music [9]. In other words, slow music is more likely to arouse people quiet, sentimental emotional state and some deep solemn feeling [10]. And fast music triggers the excitement and pleasure related to emotional state. In the business world, also scholars are concerned about the impact of speed of the music on consumer sentiment. For example, scholars Oakes proposed that Music rate will have an impact on the waiting time consumer in the waiting team, where slow music adds a positive emotional response.

\section{EXPERIMENTAL METHODS AND RESULTS}

\subsection{Experimental Methods}

The 180 subjects were randomly selected. The methods of the study were the scene of random experimentation field experiments. In this article the survey places is a university restaurant. Under the help of restaurant manager and assistant, we trained our laboratory assistant (internship attendant). In the data collection process, the author has been in the field.

\subsection{Data Processing Method}

\subsubsection{Mean}

In mathematics, mean has several different definitions depending on the context. In probability and statistics, mean and expected value are used synonymously to refer to one measure of the central tendency either of a probability distribution or of the random variable characterized by that distribution. In the case of a discrete probability distribution of a random variable $\mathrm{X}$, the mean is equal to the sum over every possible value weighted by the probability of that value; that is, it is computed by taking the product of each possible value $\mathrm{x}$ of $\mathrm{X}$ and its probability $\mathrm{P}(\mathrm{x})$, and then adding all these products together, giving

$$
\mu=\sum x P(\mathrm{x})
$$

\subsubsection{Standard Deviation}

In statistics, the standard deviation (SD) (represented by the Greek letter sigma, $\sigma$ ) is a measure that is used to quantify the amount of variation or dispersion of a set of data values.

Let $X$ be a random variable with mean value $\mu$ :

$E[X]=\mu$

Here the operator $\mathrm{E}$ denotes the average or expected value of $\mathrm{X}$. Then the standard deviation of $\mathrm{X}$ is the quantity.

$$
\begin{aligned}
\sigma & =\sqrt{E\left[(\mathrm{X}-\mu)^{2}\right]}=\sqrt{E\left[\mathrm{X}^{2}\right]+E[-2 \mu X]+E\left[\mu^{2}\right]} \\
& =\sqrt{E\left[\mathrm{X}^{2}\right]-2 \mu E[X]+E\left[\mu^{2}\right]} \\
& =\sqrt{E\left[\mathrm{X}^{2}\right]-2 \mu^{2}+\mu^{2}}=\sqrt{E\left[\mathrm{X}^{2}\right]-\mu^{2}} \\
& =\sqrt{E\left[\mathrm{X}^{2}\right]-\left(E[\mathrm{X})^{2}\right.}
\end{aligned}
$$

Standard error estimates formula is as follow:

$$
\begin{aligned}
& \sigma_{s}=\sqrt{\frac{1}{n-1} \sum_{j=1}^{n} V_{j}^{2}} \\
& \sigma=\sqrt{\frac{1}{n} \sum_{j=1}^{n} \delta^{2}}
\end{aligned}
$$

\subsection{The Results}

The experimental statistical result is shown in Table $\mathbf{1}$.

For breakfast (Fig. 1), the mean dining time of the slowpaced group is greater than the customer dining time of no music group, and is obviously greater than the customer dining time of fast- paced music group. The mean dining time of the fast-paced group is obviously less than the customer dining time of no music group. The difference between the maximum and the minimum customers dinner time is varying greatly, which is 21.1 under no music, 28.7 under slowpaced music and 22.3 under fast-paced music.

For dinner (Fig. 2), the mean dining time of the slowpaced group is greater than the customer dining time of no music group, and is obviously greater than the customer dining time of fast-paced music group too. The mean dining time of the fast-paced group is obviously less than the customer dining time of no music group with $2.7 \mathrm{~min}$. The difference between the maximum and the minimum customers dinner time is varying greatly, which is 28.3 under no music, 27.1 under slow-paced music and 23.2 under fast-paced music.

For lunch (Fig. 3), the mean dining time of the slowpaced group is greater than the customer dining time of no music group with $6.7 \mathrm{~min}$, and is obviously greater than the 


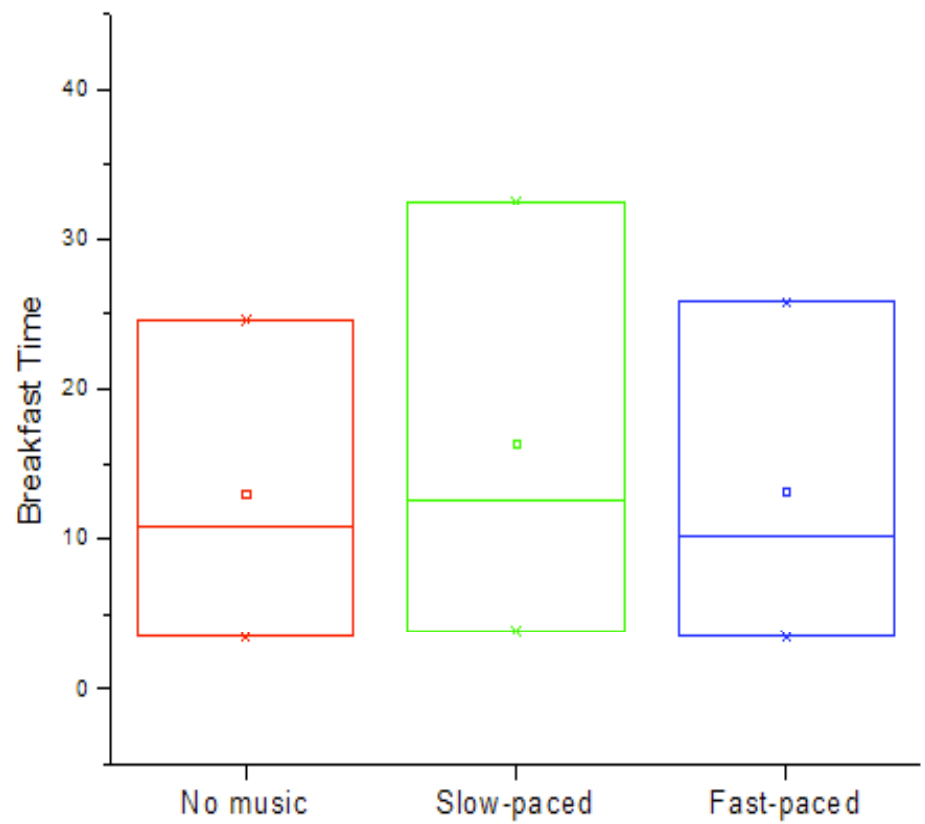

Fig. (1). The breakfast time under different types of music.

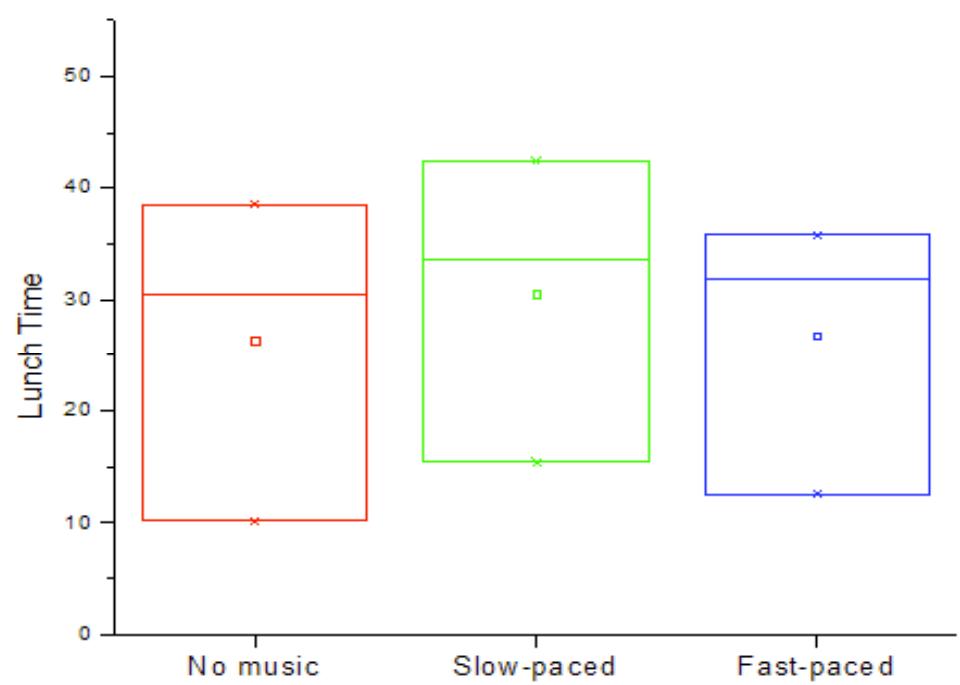

Fig. (2). The lunch time under different types of music.

Table 1. Customer dining time of breakfast, lunch and dinner under different types of music.

\begin{tabular}{|c|c|c|c|c|c|c|}
\hline Time & Music Type & Customers Number & Min & Max & Mean & Standard Deviation \\
\hline \hline Breakfast & No music & 20 & 3.5 & 24.6 & 10.8 & 1.46 \\
\hline & Slow-paced & 20 & 3.8 & 32.5 & 2.6 \\
\hline & Fast-paced & 20 & 3.5 & 25.8 & 10.2 & 30.4 \\
\hline Lunch & No music & 20 & 10.2 & 38.5 & 33.6 & 2.54 \\
\hline & Slow-paced & 20 & 15.4 & 42.5 & 35.8 & 3.8 \\
\hline Dinner & Fast-paced & 20 & 12.6 & 40.6 & 3.8 & 3.8 \\
\hline & No music & 20 & 15.6 & 52.5 & 42.5 & 3.5 \\
\hline
\end{tabular}




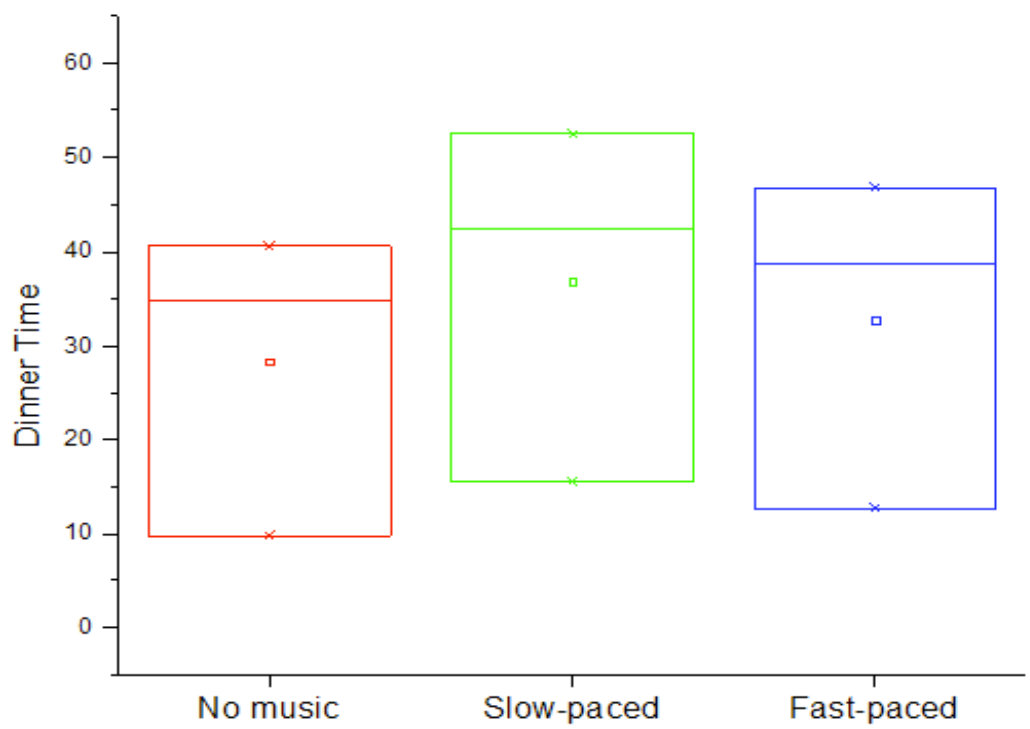

Fig. (3). The dinner time under different types of music.
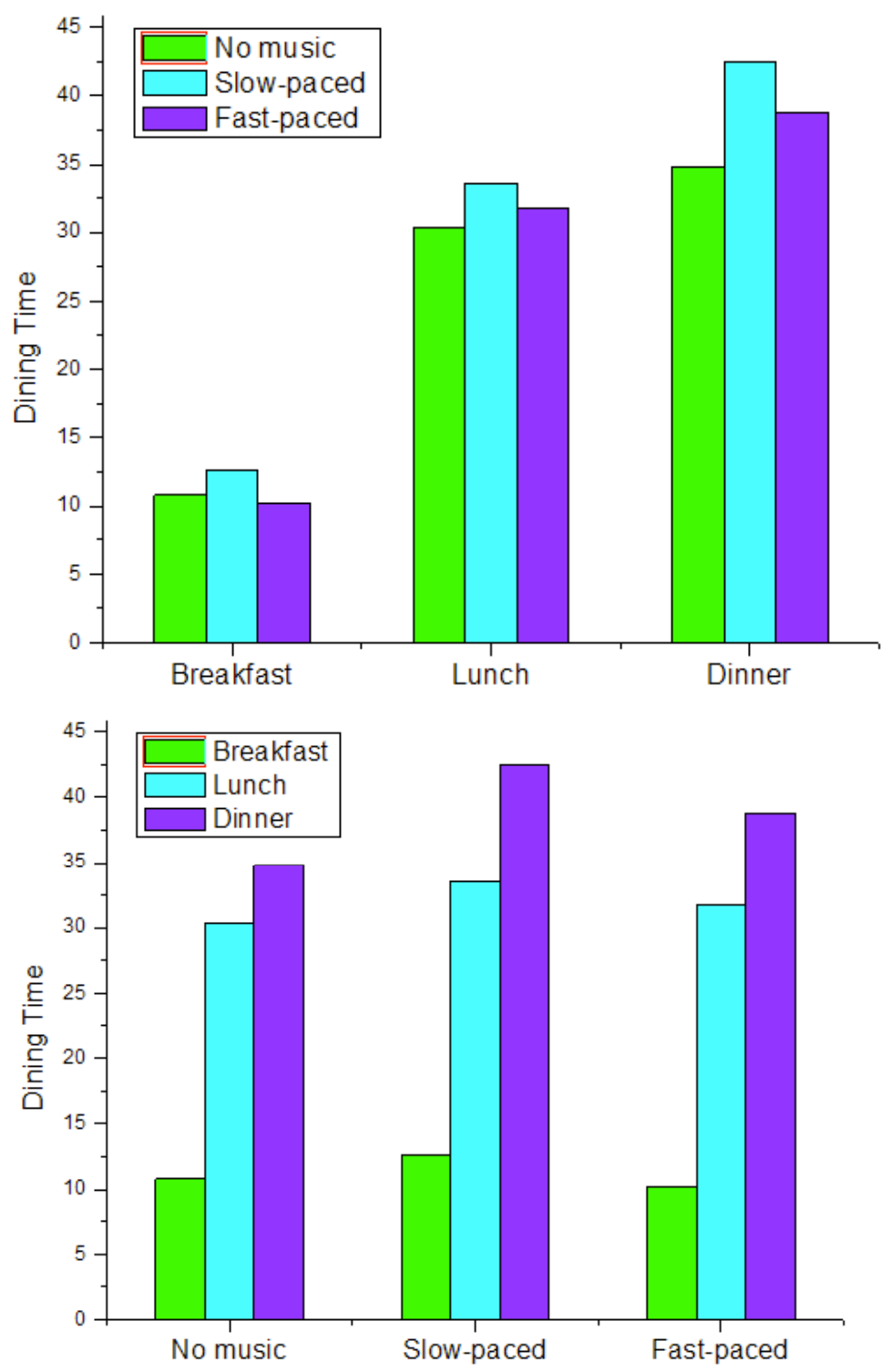

Fig. (4). The comparison results of different dinner time (left) and different types of music (right). 
customer dining time of fast- paced music group with $3.7 \mathrm{~min}$ too. The mean dining time of the fast-paced group is obviously greater than the customer dining time of no music group with $4 \mathrm{~min}$. The difference between the maximum and the minimum customers dinner time is varying greatly too, which is 30.8 under no music, 36.9 under slow-paced music and 34.1 under fast-paced music.

Further, as shown in Fig. (4), regardless of no music, slow-paced music or fast-paced music, breakfast time is much less than the lunch time and dinner time. Of three the dinner time is the longest. This is consistent with common sense: after work customers have enough time to eat and take a test.

On the other hand, regardless of breakfast, lunch and dinner, the dining time of slow-paced music is the longest. And the dining time of no music group and fast-paced music group has no statistical differences.

\section{CONCLUSION}

In this paper, we used experimental methods to study the impact of the background music on customer dining time. During the experiment, the researcher himself at the scene led and supervised the experimental data recorder recording data, minimizing the error data collected. And in the data analysis, we excluded cases with missing values. Therefore, the conclusions of this study have internal validity. The experiment was done in the restaurant around school. The restaurant has the following characteristics: Located within the school; Source to students, school teachers and their families, supplemented by outside sources; better environment than the school canteens. Other school restaurants are generally consistent with the experimental restaurant. Therefore, the conclusion of this study has reference significance to other restaurant above with an external validity.

Inadequacy of research: Since this study did not study the outside restaurant, therefore, only applying to the school restaurant, which has a small range. Experiment of this type is randomized experimental in scene, controlling experiments with some difficulty and the data collected will have a certain bias.

\section{CONFLICT OF INTEREST}

The author confirms that this article content has no conflict of interest.

\section{ACKNOWLEDGEMENTS}

This work is supported by the Key Project of Guangxi Social Sciences, China (No. gxsk201424), the Education Science fund of the Education Department of Guangxi, China (No. 2014JGA268), and Guangxi Office for Education Sciences Planning, China (No. 2013C108).

\section{REFERENCES}

[1] M. Beverland, "In-store musie and consumer-brand relationships: relational Transformation following experienees of (mis) fit.," Joumal of Business Research, vol. 59, no. 9, pp: 982-989, 2006.

[2] D. Laurette, B. H. Schmitt, and F. Leclerc, "Consumers' affective response to delays at different phases of a service delivery,". Journal of Applied Social Psychology, vol. 21, no. 10, pp. 810-820, 2011.

[3] S.A. Errol, K. Maehleit, and T.F. Barr, "Perceived retail crowding and shopping satisfaction: the role of shopping values, "Journal of Business Research, vol. 58, no. 8, pp. 1146-1153, 2010.

[4] G.D. Harrell, M.D. Hutt, and J.C. Anderson, "Path analysis of buyer behaviour under conditions of crowding," Journal of Marketing Research, vol. 17, no.1, pp. 45-51, 2008.

[5] J. Horne, "Subjective vs. objective time measures: A note on the perception of time in consumer behaviour," Journal of Consumer Research, vol. 11, no. 1, pp. 615-618, 2009.

[6] K. Kalinin, "Reading news from a pocket computer in abstracting environment : Effects of the tempo background music," Computers in Human Behaviour, vol. 2, no. 1, pp. 537-551, 2000.

[7] R. C. Larson, "Perspectives on queues: Social justice and the psychology of queuing," Operations Research, 35 (NovemberDecember), 2009, pp. 895-904.

[8] D. Moister, "The Psychology of Waiting in Lines," The Service Encounter: Managing Employees/customer Interaction in Service Business, 2005.

[9] B. J. Punnet, "Design field experiments for management research outside North America," International Studies of Management \& Organization, vol. 18, no. 3, pp. 44-54,1998.

[10] G. Tom, and S. Lucy, "A field study investigating the effect of waiting time on customer satisfaction," Journal of Psychology, vol., no. 6, pp. 655-660, 2011.

Received: June 10,2015

Revised: July 29, 2015

Accepted: August 15, 2015

(C) Yang Ting; Licensee Bentham Open.

This is an open access article licensed under the terms of the (https://creativecommons.org/licenses/by/4.0/legalcode), which permits unrestricted, noncommercial use, distribution and reproduction in any medium, provided the work is properly cited. 